\title{
Medical Image Compression for Telemedicine Applications
}

\author{
Huseyin Nasifoglu*1 and Osman Erogul ${ }^{1}$ \\ ${ }^{1}$ Department of Biomedical Engineering, Turkey
}

*Corresponding author: Huseyin Nasifoglu, Department of Biomedical Engineering, Turkey.
Received Date: May 14, 2019

Published Date: June 24, 2019

\begin{abstract}
Transferring medical images from one center to another is common use in telemedicine. These high-quality images stored in DICOM format require higher bandwidth for transmission and large storage space in PACS (Picture Archiving and Communication System) memory. Therefore, reducing the image size by preserving diagnostic information has become a need. In this sense, medical image compression is a technique that overcomes both transmission and storage cost by suggesting lossy and lossless compression algorithms. There are numerous compression methods developed for region-based studies generally used in the radiography, computed tomography (CT) and magnetic resonance (MR) images. In this review, information about region based medical image compression and recent studies with different approaches are expressed.
\end{abstract}

Keywords: Medical image compression; Lossy; Lossless; Region of interest; Telemedicine; PACS

\section{Review}

High resolution medical images obtained by different imaging modalities stored in PACS needs higher storage space and bandwidth because of requiring much space in memory. In this sense, compression of medical images is important for efficient use of database. The main purpose of image compression is to reduce the number of bits representing the image while preserving the image quality and the intensity level of the pixels as much as possible depending on grayscale or RGB image [1]. Since medical images also contain diagnostic information about a disease or an artifact, less or no loss of detail in terms of quality is desired while compressing the significant areas. Otherwise, there may be difficulties or misdiagnosis in the treatment planning. With the lossless image compression technique, it is possible to preserve the entire pixel data while reducing the image size. The disadvantage of this technique is that it does not gain high memory size due to the low compression performance. On the other hand, higher compression ratios can be obtained by compromising redundant data with the lossy compression technique. Loss of image quality seems to be a risky condition in terms of correct diagnosis, but it is possible to reach acceptable compression rates and control data loss by setting appropriate parameters without losing diagnostic information. Adaptive image compression (AIC) is a hybrid technique that combines both lossless and lossy image compression techniques [2,3]. When applying AIC, it is primarily necessary to detect regions of interest in order to determine which regions will be compressed as lossy or lossless. After determining the focused or noticeable regions of radiologist on graph, it is possible to sort and adaptively compress these regions by importance. If the first order region is considered as the area containing the most information for the diagnosis, lossless compression can be applied here so as to avoid loss of detail. If there are second, third, and continuing order regions of interest, it may be preferable to adaptively compress these areas with little loss. Non-ROI (Non-Region of Interest) parts can be considered as less important or healthy areas in the diagnostic sense. Therefore, higher compression ratios can be achieved by compromising more details for these regions. After applying AIC, reconstructed image should be evaluated as sufficient and acceptable by the physician in terms of diagnostic information. Thus, the compressed images are recommended to be evaluated with subjective criteria in addition to objective criteria.

The most common used objective criteria parameters for evaluating the compression performance are Compression Ratio (CR), Bits per Pixel (BPP), Mean Square Error (MSE) and the Peak Signal to Noise Ratio (PSNR). Compression ratio is defined as the 
ratio between the uncompressed and compressed size. BPP implies how many bits are stored per pixel and it desired to obtain lower BPP values while preserving the image quality in order to get higher compression performance. On the other hand, MSE and PSNR depend on the error/noise between compressed and uncompressed image. PSNR value can be directly obtained by MSE result and it is purposed to get a higher value of PSNR which means signal to noise ratio is higher. Reducing the size of data by preserving diagnostic information allows more images to be stored in the PACS database with memory saving. Moreover, the transmission of images from one center to another will be faster and easier for telemedicine applications.

It is possible to find out researches on medical image compression with adaptive or non-adaptive structure. In most studies, different anatomical regions have been discussed where the images are generally obtained by MR, CT and Computerized Radiography. In a study on pulmonary tuberculosis X-ray images, the performance of JPEG and wavelet transform based JPEG2000 compression algorithms has been investigated [4]. It is indicated that the JPEG algorithm performs better at low compression ratios. However, both algorithms are found to be useful on storing and transferring applications of X-ray images of chest region. All compressed images were also presented to specialists and subjectively evaluated. As a result of the evaluation, the compressed images were found to be excellent when compared to the original images.

Sophia P. and Anitha J. have considered the region of interest of the physician in compressing of MR images [5]. ROI has been compressed by lossless approach, while the Non-ROI is compressed as lossy. It has been indicated that higher compression rates were achieved when the ROI was small and an average compression ratio of 4.2 and $20.76 \mathrm{~dB}$ PSNR were obtained.

In a study investigating the compression of more than one ROI on peripheral arterial CT images, first order ROI was compressed as lossless, second order ROI as lossy and Non-ROI was compressed as lossy with higher ratio compared to second order ROI [6]. An average compression ratio of 3 and $39 \mathrm{~dB}$ PSNR were obtained as a result of objective evaluation. As a result of Mean Opinion Score (MOS) test in subjective evaluation, the physician evaluated the compressed images as almost identical the original ones. Researchers have also emphasized that the use of compressed images will reduce memory costs and facilitate data acquisition between units.

Raja A., Raja G., and Khan K. have researched compression of multiple ROIs at different rates in medical images [7]. In this study, JPEG XR and JPEG2000 standards have been tested while JPEG XR is found to be more successful for procedural convenience and better suited for embedded applications. In a study of CT images of colon tissue, ROI has been compressed as lossless and the remaining background compressed as lossy algorithm [8]. Motion compensated coding is preferred for compression and low bits per pixel values are obtained.
Seddiki A. and Guerchi D. proposed a hybrid model that combines JPEG-Huffman encoding with SPIHT for compression of thorax and brain images [9]. In brain MR images ROI was compressed as less lossy and Non-ROI part as more lossy. As a result, up to 0.6 BPP with 29.012 dB PSNR for brain and 0.55 BPP with $29.8 \mathrm{~dB}$ PSNR are obtained for thorax images. Although high compression ratios have been achieved, there has been no subjective evaluation to determine the extent of the diagnostic loss as visual.

The JPEG table used in the quantization step is usually applied for gray level images and can be scaled by using quality factor. In a study, researchers proposed a new quantization table for compression of angiocardiogram images [10].

Bradley J. and Erickson M.D have investigated the effects of lossy compression on medical images [11]. Researchers have suggested that compression studies in medical images can be applied in teleradiology and PACS, indicating that degradation in image can be kept at a noticeable level when reaching high compression ratios. In addition, it has been stated that medical images can be stored in memory with less storage space, which may reduce memory expenditures.

Nasifoglu et. al. studied compression of multiple ROIs in order of importance on pelvic fracture computed radiography images [12]. Proposed method detects and adaptively compresses three ROIs and background image (Non-ROI) with JPEG algorithm adaptively according to the manual contouring by the radiologist. First order ROI is defined as the most significant region in the image which involves definitely the fracture. Therefore, this ROI is compressed by lossless algorithm. Second and third order ROIs have less importance compared to the first order region, however they contain substantial information about fracture for the radiologist. Consequently, these ROIs are compressed by lossy algorithm with different ratios. According to the results, compressed images have an average value of $6.01 \mathrm{CR}, 2.79 \mathrm{BPP}, 37.61 \mathrm{~dB}$ PSNR and 31.06 $\mathrm{dB}$ SNR and preserve sufficient data diagnosis without losing any significant information in ROI.

Kaur A. and Monica G., preferred Haar Wavelet Transform for lossless compression of the ROI part on brain images [13]. Decoding of the data is done by applying Inverse Discrete Wavelet Transform (IDWT) method and up to 3.66 MSE against 0.86 MSE is obtained when compared with Discrete Cosine Transform (DCT) technique. According to the results proposed technique gives high performance in terms of computation and speed of computation is high.

Anandan P. and Sabeenian RS., have investigated a method for compression of various CT and MR images using Fast Discrete Curvelet Transform based on wrapping technique [14]. The curvelet coefficients are then quantized by vector quantization. As a next step arithmetic coding technique is used for encoding of the coefficients. Finally, reconstructed images are obtained by applying inverse curvelet transform and performance of the proposed algorithm is assessed by CR and PSNR. As a comparison, it has been 
observed that Curvelet transform worked better for both PSNR and CR compared with Wavelet Transform and DCT algorithms.

\section{Conclusion}

Compressing the whole image with lossy algorithm results with losing diagnostic information on critical areas. On the other hand, compressing completely with a lossless algorithm does not provide a remarkable compression ratio and memory space. Therefore, region-based compression has become a research area to investigate for higher compression rates and acceptable other objective criteria parameters. For this purpose, the image is generally segmented into regions, ROI and Non-ROI. ROI part involves the most significant diagnostic data and the Non-ROI part contains non-critical information by a specialist. By segmenting these regions lossless algorithm is applied to the ROI(s) and lossy algorithm to the Non-ROI parts. In this way, it is possible to obtain higher performance results while maintaining significant diagnostic data. It is important to choose compression technique and the related parameters appropriately for the purpose of the study. Thus, diagnostic information can be preserved, and loss of detail can be acceptable for radiologists. Consequently, compressed images will save data by occupying less memory by preserving the diagnostic information. In this way, the transmission of images from one center to another can be done more quickly with lower bandwidth for the telemedicine applications. Besides, compressed medical images contribute to the database management of PACS by having less memory.

\section{Acknowledgment}

None.

\section{Conflict of Interest}

No conflict of interest.

\section{References}

1. Gonzalez RC, Woods RE (2002) Digital Image Processing. (2nd Edition), Prentice Hall, Upper Saddle River, New Jersey, USA, pp. 409-518.
2. Sridhar KV (2008) Implementation of Prioritised ROI Coding for Medical Image Archiving using JPEG2000. International Conference on Signals and Electronic Systems, Poland.

3. Bharti P, Gupta S, Bhatia R (2009) Comparative Analysis of Image Compression Techniques: A Case Study on Medical Images. International Conference on Advances in Recent Technologies in Communication and Computing, USA.

4. Dodig M, Grgic S, Dodig S (2005) Quality of Chest X-ray Images Compressed using JPEG and JPEG2000. International Conference on Computer as a Tool (EUROCON), Serbia and Montenegro.

5. Sophia P, Anitha J (2014) Implementation of Region Based Medical Image Compression for Telemedicine Application. International Conference on Computational Intelligence and Computing Research (ICCIC), India.

6. Firoozbakht M, Dehmeshki J, Martini M, Ebrahimdoost Y, Amin H, Dehkordi M, Youannic A, Qanadli SD (2010) Compression of Digital Medical Images Based on Multiple Regions of Interest. Fourth International Conference on Digital Society, Netherlands Antilles.

7. Raja JA, Raja G, Khan K (2013) Selective Compression of Medical Images using Multiple Regions of Interest. Life Science Journal 10(9): 394-397.

8. Göktürk SB, Tomasi C, Girod B, Beaulieu C (2001) Medical Image Compression Based on Region of Interest with Application to Colon CT Images. Proceedings of the 23rd Annual International Conference of the IEEE Engineering in Medicine and Biology Society, Turkey.

9. Seddiki A, Guerchi D (2014) Medical Image Compression by Region of Interest based on SPIHT and Global Thresholding using Huffman Coding. Recent Advances in Electrical Engineering and Educational Technologies, Greece.

10. Onnasch DGW, Prause GPM, Ploger A (1994) Quantization table design for JPEG compression of angiocardiographic images. Computers in Cardiology 265-268.

11. Bradley J, Erickson MD (2002) Irreversible Compression of Medical Images. Journal of Digital Imaging 15(1): 5-14.

12. Nasifoglu H, Erogul O, Atac KA, Ozdemir, G (2017) Multi-Regional Adaptive Image Compression (AIC) for Hip Fractures in Pelvis Radiography. IFMBE Proceedings of the International Conference on Medical and Biological Engineering 62: 61-67.

13. Kaur A, Goyal M (2014) ROI Based Image Compression of Medical Images. International Journal of Computer Science Trends and Technology (IJCST) 2(5): 162-166.

14. Anandan A, Sabeenian RS (2016) Medical Image Compression Using Wrapping Based Fast Discrete Curvelet Transform and Arithmetic Coding. Circuits and Systems 7(8): 2059-2069. 